\title{
EVENT-RELATED POTENTIALS TO CHANGES OF RHYTHMIC UNIT: DIFFERENCES BETWEEN MUSICIANS AND NONMUSICIANS
}

\author{
Pekcan Ungan ${ }^{a}$, Türev Berki ${ }^{b}$, Nurhan Erbil ${ }^{c}$, Suha Yagcioglu ${ }^{c}$, Mehmet Yüksel ${ }^{b}$, \\ Rezzan Utkucal ${ }^{\mathrm{c}}$ \\ a Department of Biophysics, School of Medicine, Koc University, Rumelifeneri Yolu, Sariyer, 34450 Istanbul, Turkey \\ b Department of Musicology, Ankara State Conservatory, Hacettepe University, 06500 Ankara, Turkey \\ ${ }^{c}$ Department of Biophysics, Faculty of Medicine, Hacettepe University, 06100 Ankara, Turkey
}

\begin{abstract}
We investigated whether the expected differences between musicians and nonmusicians in their ability to detect a rhythm-change are reflected in their event-related potentials (ERPs) and, if reflected, how these ERP differences associate with behavioral indices. Stimuli were 3 consecutive and equally spaced drum beats followed by a rest period to form a rhythmic unit (RU). By using three different inter-beat periods, three RUs were produced. Combinations of these RUs served as the "target/standard" pairs of an oddball sequence. In four different experiments, we tried two RU-change types each with two levels of detection difficulty. ERPs were recorded from the F3, Fz, F4, Cz, and Pz scalp sites of 12 musicians and 12 nonmusicians. $\mathrm{RT}$, hit and false alarm rates were also measured. The data have shown with high statistical confidence that, associated with the musicians' better detection performance and shorter RTs, their ERP P3 to rhythm changes peaked significantly earlier and was significantly larger compared to nonmusicians. Intergroup ERP differences allowed above $90 \%$ correct classification. This study has also showed that not only violations of relatively complex musical regularities but very simple rhythmic unit alterations could lead to significant P3 differences between musicians and nonmusicians. The high accuracy of the musician/nonmusician classification based only on their P3 data strongly supported the hypothesis that sensory and/or cognitive advantage of musicians in detecting rhythm changes does reflect in their P3.
\end{abstract}

Keywords: Rhythm; Music; Expertise; ERP; P3; P300 


\section{INTRODUCTION}

Reaction time (RT) and accuracy are the most widely used dependent variables in experimental cognitive research. The fine time-course of the processes involved in the perception of an event and its further cognitive analysis can be studied, on the other hand, by recording the changes in the brain's electrical activity time-locked to that event (i.e., the event-related potential; ERP). Qualitatively different processes are reflected by qualitatively different waveforms of brain's electrical responses to the event studied. If the event of interest is a musical one, various components of the ERP elicited should allow one to study the dynamics of the neural processes associated with music perception.

In an 'oddball' experiment, in which the subject is instructed to detect and respond to an auditory target presented in a sequence of auditory stimuli, the target stimulus elicits in the brain a typical sequence of positive and negative deflections at various post-stimulus latencies. One of those is a large positive deflection called P3, which peaks between 300-500 ms after the onset of the attended event and is recorded with maximal amplitudes at a parietal scalp electrode. Although it has been difficult to elaborate its specific functional role, or to characterize the underlying neural machinery, there is general consensus that the P3 reflects the timing of cognitive processes [1]. This timing may be related to the succession and speed of mental processes $[2,3]$. The latency of the P3 should then correspond to the speed of cognitive processing and its amplitude shows the allocation of brain energy resources [4]. Two distinct components of the P3, namely P3a and P3b, which differ in their latency, scalp topography and psychological correlates have been identified [5].

Several reports have also described some "slow waves" (SW) that can be observed in the epoch following P3b, suggesting that they reflect further processing beyond the processes that underlies P3b (see [6], for a review). A common view of these reports is that the amplitude of these SWs increases as a function of task demand and/or with behavioral signs of improved processing efficacy [7]. García-Larrea and Cézanne-Bert [8] describe these parietally positive SWs as non-specific activities that signal the completion of any synchronized operation immediately following target discrimination. In more recent ERP studies, musically unexpected events have also been shown to elicit extremely late slow positive shifts with latencies up to $1500 \mathrm{~ms}$ (e.g., $[9,10])$.

Due to its above-summarized connections with cognitive processes and because it provides independent measures of stimulus evaluation time and attentional requirements $[11,12]$, the P3 should provide considerable insight into cortical processing of musical events, such as alterations in pitch, timber, rhythm, tempo, etc. Because it is expected that the extraordinary intensity of musical training might shape the brain and its functions [13-16], musicians form an ideal and interesting subject pool to study structural and functional adaptations associated with exceptional cognitive and motor performance. In line with this expectation, significant ERP differences have been reported to exist between musicians and nonmusicians in a number of studies employing musical stimuli (e.g., [17-21]). It should therefore be possible, by studying the ERP differences between musicians and nonmusicians, to make contributions to the basic knowledge about neural mechanisms related to cognitive processes. However, another outcome of such studies may be to discover some strong associations between electrophysiological and behavioral indices and to design EEG-based tests that can be used for objective assessment of pre-training musical abilities and, through follow-up studies, appropriateness of a particular musical training or education program. 
1 However, in contrast to the above mentioned expected effects of musical training or expertise on cognitive ERP components, there are a number of studies in the literature not supporting a clear effect of musical expertise on the P3. For example, in a study of Regnault et al. [10], the amplitudes of the $\mathrm{P} 3$ responses of musicians and nonmusicians to mismatches in consonance or harmonic context of chords were not found to be significantly different from each other. Similarly, Koelsch et al. [22] found that the P3s elicited in musical experts by changes in instrument or timbre were not significantly different from those elicited in novices, although the P3 amplitudes of experts tended to be larger. Jongsma et al. [23] reported that, although rhythm experts showed less latency-jitter of the omission-evoked positive deflection compared with nonmusicians, no group differences were observed between the amplitudes or latencies of what they called P3-like positive deflection peaking at around post-stimulus 350 ms. In another study investigating the effect of rhythm-induced expectancies on the P3 of rhythmically trained and untrained subjects, it was reported that they found no significant amplitude difference between P3s of the two groups [24].

It seems further studies on the ERP effects of musical expertise are needed to clarify the discrepancy indicated by the brief literature survey above. This has been a source of motivation for us to undertake the present study. Another motive for the present study has been the fact that the studies attempting to investigate possible musician/nonmusician differences of ERPs related to rhythm perception are relatively scarce [21, 24-27]. Furthermore, in these handful of studies either negative results were reported about the presence of a P3 difference, or the events presented to their subjects were not actual rhythm changes. The first study in this short list demonstrates the effect of "subjective accenting" on the ERPs evoked by loudness deviances, but without an actual change in the rhythm of isochronous tone sequences. The second one deals only with the MMN (mismatch negativity), which is known to be a pre-attentive ERP component that reflects an automatic auditory mismatch detection mechanism [28], and not with the P3 deflection, which is believed to reflect the level of attention, speed of information-processing and capacity related processes. In the next two studies comparing musicians with nonmusicians, Jongsma et al. $[23,27]$ found some ERP differences such as less latency variability of omission evoked potentials, and emergence of an N600 in their responses to expectedly omitted stimuli in musicians; they observed, however, no difference between the P3s of the two groups. In the next study, where Jongsma et al. [21] investigated the P3s elicited by small accelerations and decelerations of tempo, the P3 did show main effects of group. However, neither group consisted of professional musicians; both groups were ad-hoc recruited students. In the last one of the above listed studies [24], in which the effect of rhythm-induced expectancies on the P3 was investigated, the authors reported that they could observe no significant P3 amplitude or latency advantage for the rhythmically trained group. In brief, we could find in the literature no study indicating a clear musician/nonmusician difference in their ERP P3s evoked by actual changes in rhythm.

Because of the above mentioned rather divergent findings about the effects of musical training/ expertise on the $\mathrm{P} 3$ and because of the relative scarcity of studies on possible musician/nonmusician differences of ERPs related to rhythm perception, we undertook the present study to compare the ERP P3s recorded from musicians and nonmusicians when they respond to changes of rhythmic unit (RU).

According to ANSI (American National Standards Institute) acoustical terminology, a rhythm is a temporally patterned sequence of durations and may have various levels of complexity. A rhythm can be formed by grouping of musical sounds, principally by means of duration and stress [29]. Comprehension of the rhythmic structure in any work of music depends on the 
repetition of the "grouping" in certain order. Thus for the perception of the rhythm, rhythmic units which are repeated many times are essential. It is known that when listeners hear a regular sequence of identical sounds, they tend to group them by twos, fours, or threes [30]. Since nothing objectively specifies the grouping, this kind of rhythmization is subjective. To avoid the interindividual variance which might thus have resulted, we had to introduce some irregularity into the sequence of sounds. For instance, one element might have been longer, louder, a different pitch, or followed by a longer pause [30]. We preferred the last one of these alternatives to avoid complexities which would have arisen from possible interactions with other musical dimensions such as melody and harmony [31]. We believe this choice has helped us avoid excessive and difficult-to-interpret inter-individual ERP variations that would otherwise occur due to different strategies the subjects might use in processing a complex temporal pattern and due to different melodic associations it might invoke.

\section{METHODS}

16

\section{Subjects:}

14 musicians (students and graduates of conservatory with at least 5 years of music training and/or playing an instrument; mean age 21.4; range 16-26; 8 males) and 12 nonmusicians (university students with no history of a hearing problem who have neither received any formal musical training nor played any instrument; mean age 22; range 19-25; 6 males) were studied. Data of 2 musicians (male) were discarded because of continuous and excessive artifacts. Considering possible age dependencies of ERP latencies, age ranges of the two groups were balanced. Musician subjects were unpaid volunteers displaying already a high motivation to participate in the experiments. Nonmusician volunteers were paid to maintain a motivational balance with the musician group. Written consents of the subjects in both groups were obtained and the project was approved by the ethics committee of Hacettepe University.

\section{rhythmic units}

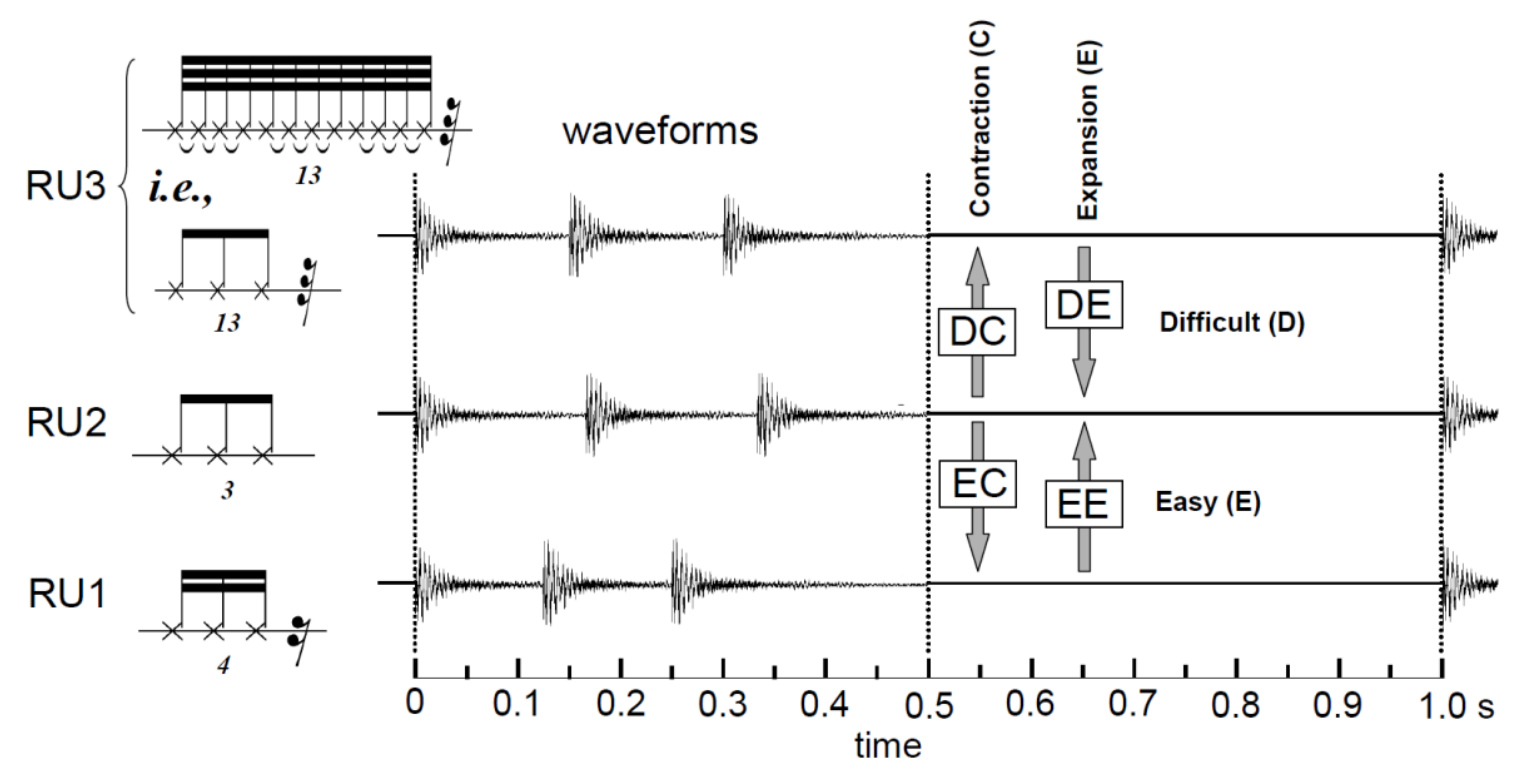

Figure 1. The three rhythmic units (RU1, RU2, RU3) used to form the standard/target stimulus pairs in the four oddball experiments carried out. The waveforms of these rhythmic units are also given. Vertical axis is the sound amplitude in arbitrary units. Depending on whether RU1 or RU3 is paired with RU2 to form the deviance, targets with two detection difficulty levels (easy and difficult, respectively) are created. And, for each difficulty level, two different targets with 
opposite change directions (contraction and expansion) are formed. This resulted in the following four different target changes: Easy-to-detect contraction $(\boldsymbol{E C})$, Easy-to-detect expansion $(\boldsymbol{E E})$, Difficult-to-detect contraction $(\boldsymbol{D C})$, Difficult-to-detect expansion $(\boldsymbol{D E})$.

\section{Stimulation:}

Three different rhythmic units, each containing 3 consecutive and isochronous drum beats in a unit time of $500 \mathrm{~ms}$ were synthesized by Soundforge 8.0 software (Sony Creative Software, Madison, WI) and labeled as RU1, RU2 and RU3. In RU1, unit time is divided into four equal intervals and first three of them are played. In RU2, unit time is divided into three equal intervals and all of them are played. In RU3, unit time is divided into thirteen equal intervals and among them, the first, the fifth and the ninth are played. These RUs and their waveforms are presented in Fig. 1. A rhythmic sequence was formed such that a three beat-RU followed by a rest period of around $500 \mathrm{~ms}$ was repeated at a rate of $1 / \mathrm{sec}(60$ beats $/ \mathrm{min})$. As expected, the first beat of the RU following the rest period served as the accenting sound and maintained objective rhythmization. This was also in line with the finding of Garner [32] who observed that the beginning of the finger tapping response pattern tended to coincide with the beginning of the longest run of similar auditory elements, when the subjects tried to reproduce a particular sound pattern.

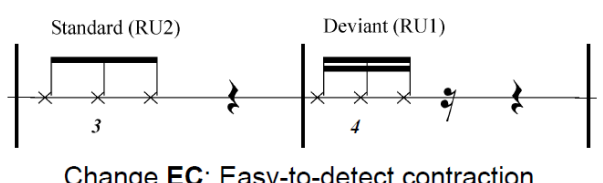

Change EC: Easy-to-detect contraction

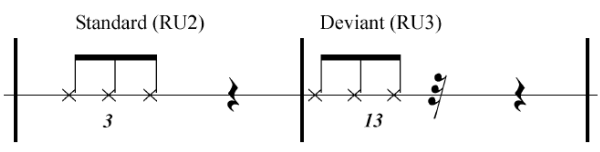

Change DC: Difficult-to-detect contraction

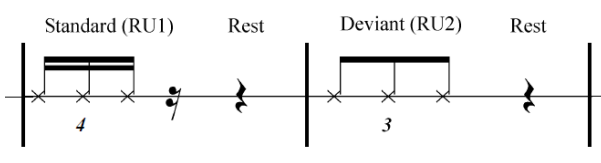

Change EE: Easy-to-detect expansion

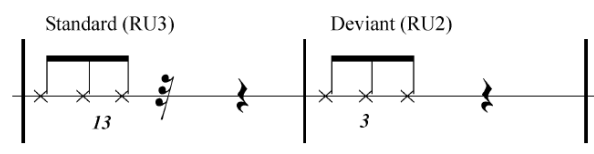

Change DE: Difficult-to-detect expansion

Figure 2. The rhythmic unit changes $(\boldsymbol{E C}, \boldsymbol{E E}, \boldsymbol{D C}$, and $\boldsymbol{D E})$ that were produced during standard-to-deviant transitions in the four oddball experiments carried out.

The three RUs were combined pairwise to form the "standard / target" pairs of an oddball sequence in four different ways. Occasional occurrence of a target after a series of standards produced a change in RU. These changes were made in either directions which may be called "contraction" and "expansion". Depending on the direction and magnitude of the difference between the inter-beat intervals in the standard and target units, RU-changes in two directions (contraction and expansion) each with two levels of detection difficulty (easy and difficult) were obtained by filling the standards and targets of the oddball sequence in four different ways labeled EC, EE, DC, and DE as shown in Fig. 2.

RU2, which divides the unit time into three equal parts, is called a triplet and widely used in music. It was therefore given a central role in designing the RU-changes and employed in all of the "frequent/rare" RU pairs forming the changes that were used as the target event. In the easyto-detect changes RU2 was paired with RU1, which divided the unit time into four equal parts. In the difficult-to-detect changes, however, RU2 was paired with RU3, forming a couple of which the members could not easily be differentiated from each other. The difference between the inter-beat intervals in RU2 and RU3 was only 8\%. This change corresponded to a time difference that was shown to be barely perceivable [33]. 
The number of standard stimuli between two successive targets was randomized between 3 and 9, corresponding to an average target-to-target interval (TTI) of 7 seconds and to a deviant/ standard proportion of 1 to 6 (i.e., 14\% of the stimuli were deviants). The selected tempo of 60 beats/min corresponded to a stimulus onset asynchrony (SOA) of 1 second. Waveforms obtained as wav files were loaded to Neuroscan's Stim1 system (Compumedics, Charlotte, NC, USA) and presented at an intensity of $90 \mathrm{~dB}$ via a loudspeaker placed in front of the subject at a distance of $1 \mathrm{~m}$. Considering the slow stimulation rates generally employed in P3 studies, a SOA of 1 second may seem relatively short for recording this ERP component. However, it is reported that target-to-target interval (TTI) and not inter-stimulus interval (ISI) is a critical determinant of P3 responsivity [34]. Their data show that there may not be much difference between the P3 amplitudes recorded with ISIs of 1 and 2 seconds, provided that a target is preceded by at least three standard stimuli. This condition is satisfied in our present study. Croft et al. [35] showed that P3 amplitude increases and its latency decreases linearly with TTI, and these relations are relatively unaffected by ISI. Acording to their results, a TTI around $7 \mathrm{~s}$ should be optimum in the sense that increasing TTI from $7 \mathrm{~s}$ to $10 \mathrm{~s}$ would result in an amplitude enhancement of only $10 \%$ (with no latency-change) but at an expense of $40 \%$ increase in recording time. The average TTI used in the present study should thus be considered an optimal choice.

\section{Recording procedures:}

Subjects were allowed to listen to these stimuli just before the recording session to familiarize with the rhythms and have some practice detecting their occasional changes. They were then instructed that during recordings they should press a button as soon as they detect a target RU and refrain from responding to standard RUs. In each of the four sessions run with the abovedescribed combinations of two discrimination difficulty levels (easy and difficult) and two types of change (contraction and expansion), EEG was continuously recorded to include at least 96 artifact-free responses to targets. In order to minimize the risk of ERP habituation, each session was divided into six successive blocks of 16 targets separated by relaxation breaks of around one minute between them. The first and second sessions were always with easy-to-detect changes (either $\mathbf{E C}$ or EE, randomized); the third and fourth sessions were always with difficult-to-detect changes (either with DC or DE, also randomized).

EEG was registered with tin electrodes fixed in an elastic electrode cap (Electrocap International, Eaton, Ohio, USA) according to the 10-20 electrode system (American Encephalographic Society, 1994). Recording was made with vertex (Cz) reference from the electrodes F3, Fz, F4, Pz, and two extra electrodes attached to earlobes to be used for rereferencing. The ground electrode was on the forehead. Electrode impedances were kept below $10 \mathrm{k} \Omega$. Stimulus onset and button-press times were recorded simultaneously with continuous EEG, by using a Nuamps amplifier run under Neuroscan software (Compumedics, Charlotte, NC, USA). Passband of the online filters and artifact rejection were set to .05-70 Hz (two cascaded $2^{\text {nd }}$ degree Butterworth) and $\pm 150 \mu \mathrm{V}$, respectively. EEG was sampled at $250 \mathrm{~Hz}$.

\section{Data processing:}

Offline, the data were digitally low-pass filtered (bidirectional $2^{\text {nd }}$ degree $30 \mathrm{~Hz}$ Butterworth). EEG traces were then epoched between pre-stimulus $100 \mathrm{~ms}$ and post-stimulus 2,000 ms ("stimulus" is the first drum beat of the RU). The waveforms were also analyzed offline by using digital high-pass filtering (bidirectional $2^{\text {nd }}$ degree Butterworth, $1 \mathrm{~Hz}$ ) to remove the effect of the positive slow wave (PSW) on P3 amplitude. $1 \mathrm{~Hz}$ cut-off frequency was selected as the logarithmic half-way between the approximate main frequencies of the PSW and P3, which were roughly estimated to be $.5 \mathrm{~Hz}$ and $2 \mathrm{~Hz}$, respectively, from the half-wave periods of these waves (i.e., $1 \mathrm{~s}$ and $250 \mathrm{~ms}$ ). Using high-pass filtered EEG for measuring the amplitude and latency of 
the P3 should have also helped avoid complications that would be brought about due to EEG epochs with non-zero mean. To obtain the average ERP waveforms, around 90 peri-stimulus epochs of the responses to targets and around 550 peri-stimulus epochs of the responses to standards recorded in each experiment were averaged separately. Those $2.1 \mathrm{sec}$-epochs of standard responses overlapping in their later half with a target stimulus were excluded from averaging.

Average ERPs were digitally converted to bi-auricular reference. To obtain the endogenous ERP components, the responses to standards were subtracted from those to targets evoked by the physically same stimuli but recorded in a different session. For example, to obtain the difference-waveform with only the endogenous components of the ERP to easily detected contraction-type change, the responses to standards in change $\mathbf{E E}$ were subtracted from the responses to targets in change $\mathbf{E C}$.

The latency of the P3 was measured with reference to the time of the "event" that elicits this component, which is related to the timing of the "second" drum beat of the RUs. P3 peak latency was defined as the "post-event" time at which the $1 \mathrm{~Hz}$-high-pass filtered ERP at Pz had maximum positivity within the post-stimulus periods of $420-580 \mathrm{~ms}$ and $500-650 \mathrm{~ms}$ for the experiments with easy-to-detect and difficult-to-detect RU changes (changes EC, EE, and changes DC, DE), respectively. The amplitude at this latency was taken as the P3 amplitude. Amplitude of the positive slow wave was measured as the mean amplitude of the $.05-30 \mathrm{~Hz}$ band-pass filtered difference-ERP within the post-stimulus range of 720-1320 ms for the changes EC, EE, and 850-1450 ms for the changes DC, DE. It should be noted that these "poststimulus" time periods are to be converted to "post-event" latency ranges by subtracting the time between stimulus onset and the event of interest; i.e., $125 \mathrm{~ms}$ for the changes EC, EE, $154 \mathrm{~ms}$ for the changes DC, DE. Using the mean amplitude of the $100 \mathrm{~ms}$ pre-stimulus period (preceding the first drum beat), baseline correction was made before amplitude measurements.

Post-event average RTs as well as the hit and false alarm rates were determined for each subject. A button press to a stimulus was evaluated as a hit or false alarm if it occurred within the $1 \mathrm{sec}$ time period between that stimulus and the next one. d-prime was calculated as the difference between the z-scores of the hit and false-alarm rates [36] and used as an index for performance of detecting the target.

\section{Statistics}

eparate three-way ANOVAs for repeated measures were run on the measured differences in P3 amplitude and latency, amplitude of the positive slow wave, RT, and d-prime values. The between-subjects effect tested was GROUP (musicians and nonmusicians), the within-subject effects tested as repeated measures were DIFFICULTY of discrimination (easy and difficult), and type of CHANGE (contraction and expansion). P3 and PSW amplitudes measured at Fz, Cz, and $\mathrm{Pz}$ were subjected to ANOVA for a difference between the midline topographies of these waves. Greenhouse-Geisser corrections were employed where applicable. Post hoc evaluations were conducted by two-tailed t-tests (paired, for within-subject comparisons). Also, discriminant analyses were performed to determine which of the parameters measured under which experimental conditions are the most important variables in an accurate discrimination between the members of the two groups, and also to see if ERP parameters allow as successful a discrimination as behavioral parameters. Correlation between RT and the P3 latency is evaluated by means of linear regression analysis applied to subject averages.

\section{RESULTS}


The grand average ERPs to standard and target RUs obtained from the musician and nonmusician groups in four different main experiments are presented in Fig. 3. The ERPs recorded from the left and right frontal electrodes (F3 and F4) were very similar to those recorded from the midline frontal electrode $(\mathrm{Fz})$. Furthermore, none of the deflections mentioned above displayed any notable hemispheric asymmetry. Therefore, the ERPs obtained from F3 and F4 are not given in figures to save space. Please note that, in this and the following figures, the waveforms are presented with two different time axes for the "post-stimulus" and "post-event" times, as explained in the legend to Fig. 3.

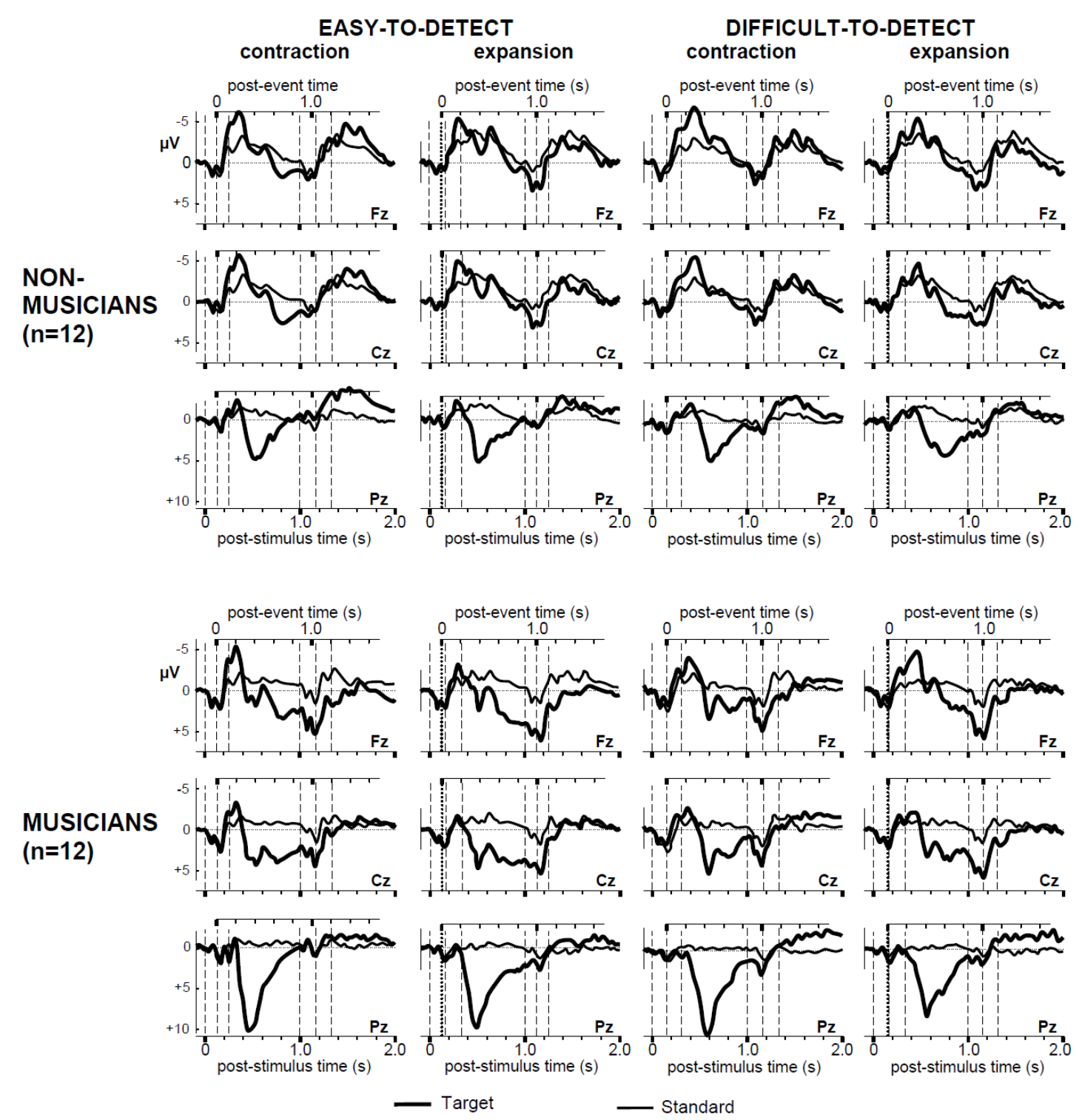

\section{ERP data}

Figure 3. Grand average ERP waveforms recorded from twelve musicians to four different rhythmic unit (RU) changes (lower panel) and from twelve nonmusicians to the same rhythmic unit $(R U)$ changes (upper panel). The potentials recorded between $C z$ and the midline electrodes at $\mathrm{Pz}, \mathrm{Cz}$, and $\mathrm{Fz}$ were re-referenced off-line to averaged earlobes. The ERP pairs presented in each column were used to obtain the "target-standard" difference-potential traces presented in Fig 4. Please note that, for the ERPs to RU-changes, the target-and standard-evoked potentials paired in a column were recorded in different experimental sessions with physically identical 
stimuli, as explained in Methods. The traces are aligned with respect to the average amplitude in a 100-msec-long pre-stimulus baseline. The zero for the post-stimulus time shows the onset of the first drum beat in the RU. Onsets of the three drum beats in a RU are also indicated by dashed vertical lines. The zero for the post-event time, on the other hand, is defined as follows for the different types of changes used. Contraction in RU: The onset of the second beat in the target $R U$, which occurs earlier than its expected time regarding the standards. Expansion in $R U:$ The onset of the second beat of the standard $R U$, which is the time the second beat is implied to occur (by the frequent standards), but delayed.
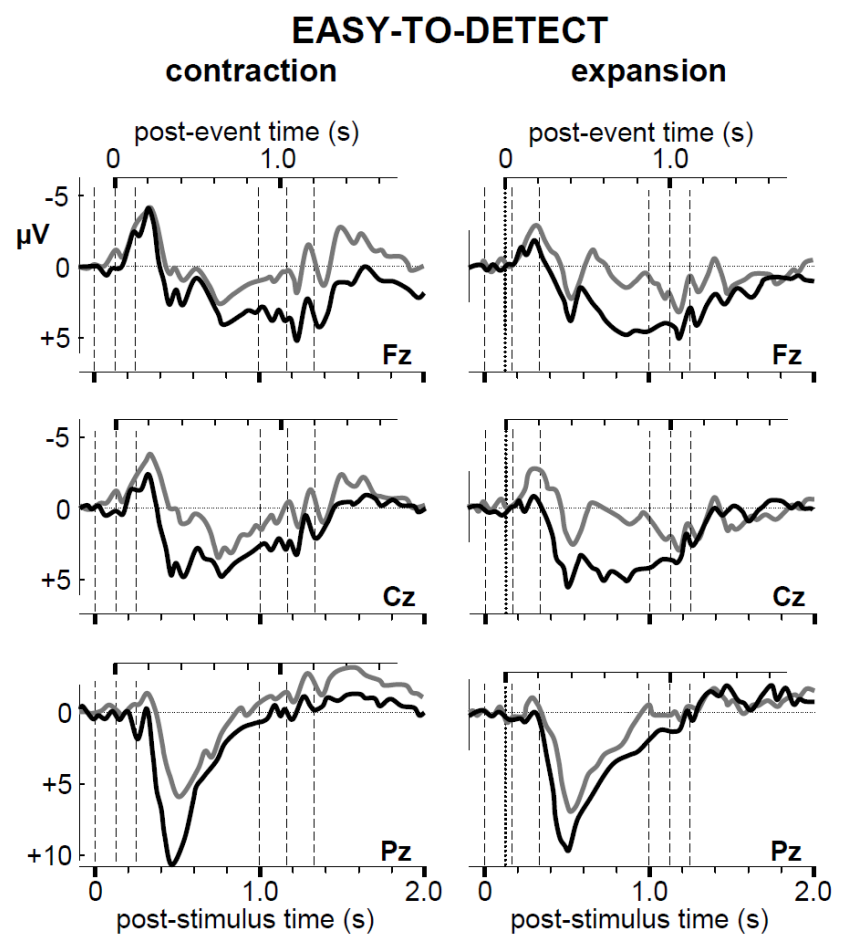

Musicians

\section{DIFFICULT-TO-DETECT contraction}
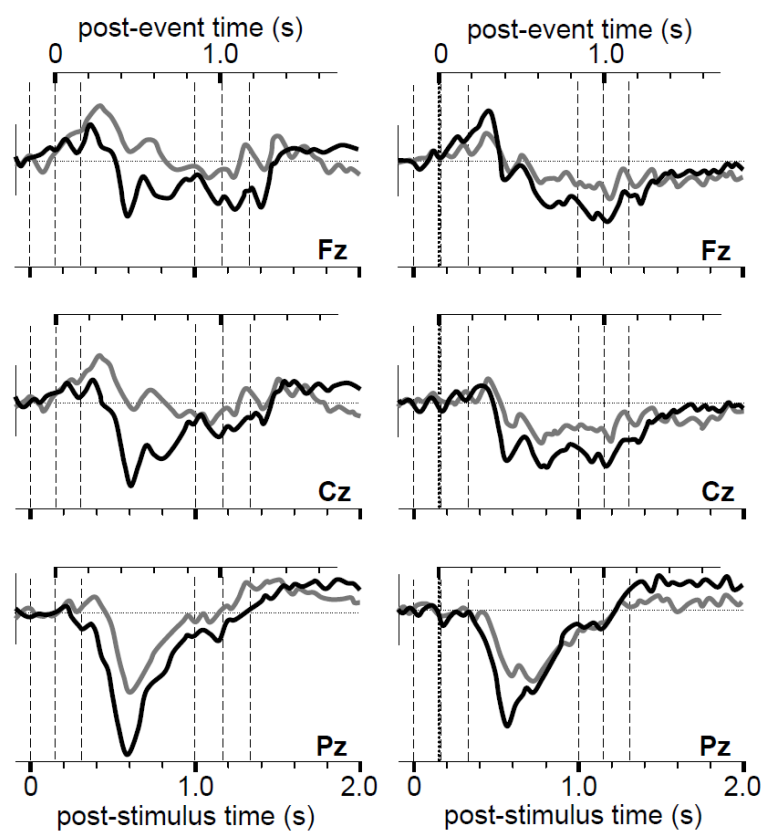

Nonmusicians

Figure 4. Grand average "target-standard" difference-potential waveforms obtained from the ERPs to RU-changes presented in Fig 3. For the post-event timing details see the legend to Fig. 3. 


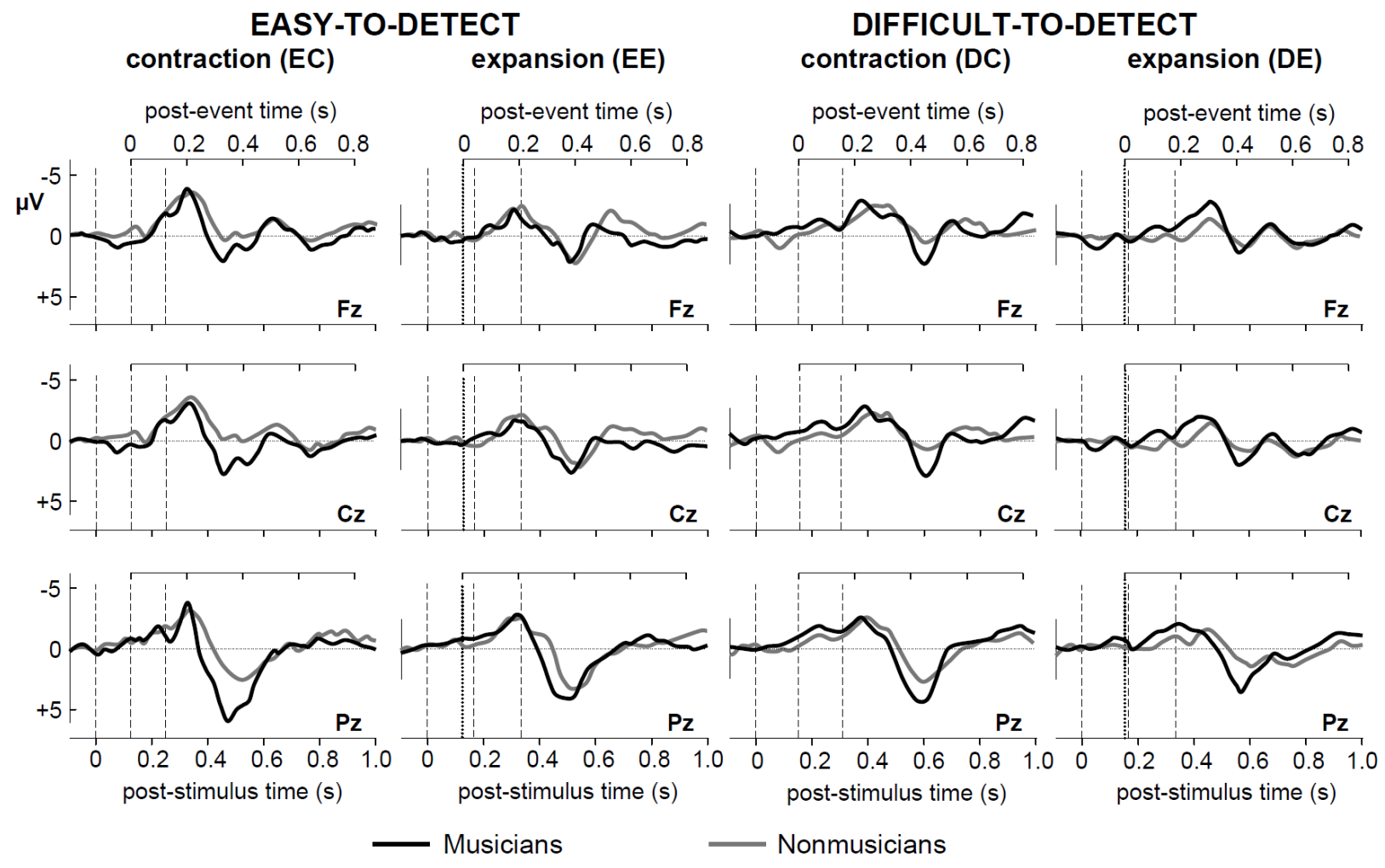

Figure 5. The $1 \mathrm{~Hz}$-high-pass filtered components of the waveforms in Fig. 4. For timing details see the legend to Fig. 3.

The ERPs to standard RUs generally consisted of a superposition of the typical P1-N1-P2 deflections to each of the three transient sounds (i.e., drum beats) in a RU and these peaks appear as undulations on a relatively slow and fairly steady negative wave. The ERPs to target RUs, on the other hand, had some additional components with negative peaks or negative-going deflections within the post-event latency ranges of $150-300 \mathrm{~ms}$ and $400-600 \mathrm{~ms}$, and a positive peak within the post-event latency range of $350-450 \mathrm{~ms}$, followed by a very slowly changing positive deflection extending in some cases even beyond $1000 \mathrm{~ms}$. Since we are particularly interested in the endogenous ERP components in the present study, the above mentioned deflections with relatively shorter latencies will not be described further.

The difference-potential waveforms presented in Fig. 4 are the endogenous components of the RU-change ERPs presented in Fig. 3. These difference-ERPs consist of some relatively early and fast phasic components and a late and almost sustained deflection called PSW (positive slow wave). To facilitate the evaluation of the phasic ERP components by reducing the confounding effect of this slow deflection, the difference waveforms were $1 \mathrm{~Hz}$ high-pass filtered as explained in Methods. The phasic components isolated in this way are given in Fig. 5.

In all the difference-waveforms presented in Fig. 4, a parietally dominant P3 is present within the post-event 350-450 ms latency range, and its baseline-referenced amplitude seems to be larger in musicians than nonmusicians. The P3 continues to be the most robust endogenous response component even after the positive slow wave (PSW) superimposed on it is largely eliminated by 1Hz-high-pass filtering (Fig. 5). Also in these filtered waveforms, a substantial amplitude difference is observed between the parietal P3s of the two groups except with the difficult-to-detect contraction target. In the cases of 'Easy-Contraction' and 'DifficultExpansion', the P3 of musicians appears to have a peak-amplitude about twice as large as those of nonmusicians. 
1 Of the various endogenous components seen in Fig 4, only the P3 and PSW, which appeared to display the most prominent, robust and repeatable differences between the two groups, were statistically evaluated in detail. Mean values and standard deviations of the parameters associated with these components that were measured in musicians and nonmusicians for two detection difficulty levels and two change directions are documented in Table $\mathbf{1 .}$

Table 1. Mean values (bold) and standard deviations (in parenthesis) of the ERP parameters evaluated.

\begin{tabular}{|c|c|c|c|c|c|}
\hline \multicolumn{2}{|c|}{ Experiment } & \multirow[b]{2}{*}{ Group } & \multirow{2}{*}{$\begin{array}{l}\text { P3 latency } \\
\text { (ms) }\end{array}$} & \multirow{2}{*}{$\begin{array}{l}\text { P3 ampltd. } \\
\text { at } \mathrm{Pz}(\mu \mathrm{V})\end{array}$} & \multirow{2}{*}{$\begin{array}{c}\text { PSW ampltd. } \\
\text { at Fz }(\mu \mathrm{V})\end{array}$} \\
\hline Difficulty & Change & & & & \\
\hline \multirow{4}{*}{ Easy } & \multirow{2}{*}{ Contraction } & Nonmusician & $388(63)$ & $3.5(2.1)$ & $1.1(2.5)$ \\
\hline & & Musician & $360(43)$ & $6.4(2.3)$ & $3.6(4.4)$ \\
\hline & \multirow{2}{*}{ Expansion } & Nonmusician & $412(37)$ & $4.4(2.6)$ & $1.4(4.0)$ \\
\hline & & Musician & $372(41)$ & $4.9(3.1)$ & $4.0(5.0)$ \\
\hline \multirow{4}{*}{ Difficult } & \multirow{2}{*}{ Contraction } & Nonmusician & $464(28)$ & $3.6(1.6)$ & $0.3(2.3)$ \\
\hline & & Musician & $440(29)$ & $5.2(2.4)$ & $2.5(3.4)$ \\
\hline & \multirow{2}{*}{ Expansion } & Nonmusician & $\mathbf{5 0 0}(80)$ & $1.9(1.5)$ & $1.3(2.5)$ \\
\hline & & Musician & 412 (33) & $4.0(2.4)$ & $2.9(3.5)$ \\
\hline
\end{tabular}

Statistics of these ERP parameters are graphically presented in the upper row of Fig. 6 together with the results of the ANOVAs which were run separately on each of them. The P3 was around $50 \%$ larger $[\mathrm{F}(1,22)=6.16, \mathrm{p}=.021]$ and peaked $45 \mathrm{~ms}$ earlier $[\mathrm{F}(1,22)=14.4, \mathrm{p}=.001]$ in musicians than nonmusicians. Across both groups, its amplitude was significantly greater for easy-to-detect RU-changes than difficult-to-detect ones $[\mathrm{F}(1,22)=11.395, \mathrm{p}=.003]$. Separate ANOVAs that were run for each group indicated that differentiation difficulty was a significant main effect on the P3 amplitude in musicians $[\mathrm{F}(1,11)=5.419, \mathrm{p}=.04]$ as well as in nonmusicians $[\mathrm{F}(1,11)=5.984, \mathrm{p}<.032]$. However, a significant difficulty $\times$ change-direction interaction in nonmusicians $[\mathrm{F}(1,11)=9.748, \mathrm{p}=.010]$ indicated that the significance of this main effect in this group is confined to expansion type of changes. RU-changes of contraction type proved to evoke greater $\mathrm{P} 3 \mathrm{~s}[\mathrm{~F}(1,22)=5.35, \mathrm{p}=.030]$ than those of expansion type, except for the easy-to-detect changes perceived by nonmusicians (significant difficulty $\times$ change-direction $\times$ group interaction; $[\mathrm{F}(1,22)=5.26, \mathrm{p}=.032])$. P3 latency, however, was significantly affected only by the detection difficulty of the RU-change $[\mathrm{F}(1,22)=74.0, \mathrm{p}<.001]$; direction of the change did not prove to be significant $[\mathrm{F}(1,22)=1.35, \mathrm{p}=.258]$. Because there was a slightly significant interaction between change-direction and group $[\mathrm{F}(1,22)=4.42, \mathrm{p}=.047]$, separate ANOVAs were run for each group and the change direction was found to be a significant effect on P3 latency neither in musicians $[\mathrm{F}(1,11)<1]$ nor in nonmusicians $[\mathrm{F}(1,11)=3.78, \mathrm{p}=.078]$.

The discriminant analysis performed showed that the P3 latency measured for difficult-to-detect RU-changes of expansion type and the P3 amplitude measured for easy-to-detect RU-changes of contraction type are the first and second most important ERP parameters (with correlation sizes of .500 and -.460 , respectively, in the structure matrix) in differentiating between the members of the two groups. Using the first parameter alone, correct classification percentage was $75 \%$ (Wilks' $\lambda=.632 ; \chi^{2}=9.872, \mathrm{df}=1, \mathrm{p}=.002$ ). Inclusion of both amplitude and latency values of the $\mathrm{P} 3$ recorded in all of the four experiments raised the correct classification percentage to $91.7 \%$ (Wilks' $\lambda=.300 ; \chi^{2}=21.680, \mathrm{df}=8, \mathrm{p}=.006$ ). Details of the classification results are given in Table 2 for the two cases. 
Table 2. Classification tables showing the number of correctly and falsely predicted group memberships estimated by discriminant analysis. Numbers of correct predictions are printed in bold. Most important variable is the variable with the largest structure coefficient of the discrimination model, which proved to be the P3 latency.

\begin{tabular}{|c|c|c|c|c|c|}
\hline \multirow[b]{3}{*}{ Variable } & \multirow[b]{3}{*}{$\begin{array}{l}\text { Actual Group } \\
\text { Membership }\end{array}$} & \multicolumn{4}{|c|}{ Predicted Group Membership } \\
\hline & & \multicolumn{2}{|c|}{$\begin{array}{l}\text { With the most important } \\
\text { variable (stepwise) }\end{array}$} & \multicolumn{2}{|c|}{$\begin{array}{l}\text { With all variables } \\
\text { (non-stepwise) }\end{array}$} \\
\hline & & Nonmusician & Musician & Nonmusician & Musician \\
\hline \multirow{2}{*}{ ERP P3 } & Nonmusician & 7 & 5 & 11 & 1 \\
\hline & Musician & 1 & 11 & 1 & 11 \\
\hline \multirow{2}{*}{ Behavioral } & Nonmusician & 9 & 3 & 11 & 1 \\
\hline & Musician & 3 & 9 & 1 & 11 \\
\hline
\end{tabular}

In the target-ERPs (Fig. 3) and difference-waveforms (Fig. 4) of both groups, all the above mentioned deflections appear to be superimposed on a nearly steady positive slow wave (PSW) following the P3. This slow wave is clearly seen at parietal as well as at frontal and central electrodes. The PSW amplitude measured at the frontal electrode was about three times larger in musicians than nonmusicians and this difference proved to be close to significance $[F(1,22)=3.997, p=.058]$. Neither the differentiation difficulty nor the direction of the RU change had a significant effect on the frontal PSW amplitude ( $\mathrm{F}=1.4$ and $\mathrm{F}<1$, respectively), with no main effect interaction. A clear difference between the midline topographies of the P3 and PSW was indicated by a significant electrode $\times$ wave interaction $[F(1.814,22)=30.6, p<.001 ; \varepsilon=.907]$. Both groups displayed the same topographical difference which was most clearly seen at the parietal electrode (post-hoc t-tests: $p<.005$ and $p<.001$ for musicians and nonmusicians, respectively; Fig. 6). The P3 and PSW amplitude differences between musicians and nonmusicians were also reflected in the between-group statistics $[F(1,22)=11.1, p=.003]$ in this 3-electrode analysis.

\section{Behavioral data}

Mean values and standard deviations of the behavioral parameters (i.e., the post-event reactiontime, and the detection performance) that were measured from musicians and nonmusicians with two detection difficulty levels are given in Table 3. Statistics of these two parameters are graphically presented in the lower row of Fig. 6 together with the results of the ANOVAs which were run separately on each of them.

Table 3. Mean values (bold) and standard deviations (in parenthesis) of the behavioral parameters.

\begin{tabular}{|c|c|c|c|c|c|c|}
\hline \multicolumn{2}{|c|}{ Experiment } & \multirow[b]{2}{*}{ Group } & \multirow{2}{*}{$\begin{array}{l}\text { Reaction } \\
\text { time (ms) }\end{array}$} & \multirow{2}{*}{$\begin{array}{l}\text { Hit rate } \\
(\%)\end{array}$} & \multirow{2}{*}{$\begin{array}{l}\text { False- } \\
\text { alarm } \\
\text { rate }(\%)\end{array}$} & \multirow{2}{*}{$\begin{array}{l}\text { Detection } \\
\text { perform. (d') }\end{array}$} \\
\hline Difficulty & Change & & & & & \\
\hline \multirow{4}{*}{ Easy } & \multirow{2}{*}{ Contraction } & Nonmusician & $454(84)$ & $94.0(1.5)$ & $1.7(0.8)$ & $3.75(.39)$ \\
\hline & & Musician & $379(59)$ & $94.0(2.2)$ & $2.9(2.0)$ & $3.64(.66)$ \\
\hline & \multirow{2}{*}{ Expansion } & Nonmusician & $418(85)$ & $94.0(2.0)$ & $1.7(1.1)$ & $3.81(.56)$ \\
\hline & & Musician & $346(55)$ & $96.4(1.3)$ & $1.7(1.3)$ & $4.07(.51)$ \\
\hline \multirow{4}{*}{ Difficult } & \multirow{2}{*}{ Contraction } & Nonmusician & $537(103)$ & $70.6(11)$ & $7.1(8.0)$ & $2.29(1.1)$ \\
\hline & & Musician & $428(63)$ & $83.3(7.5)$ & $2.2(1.6)$ & $3.16(.55)$ \\
\hline & \multirow{2}{*}{ Expansion } & Nonmusician & $470(71)$ & $80.5(3.9)$ & $3.5(2.5)$ & $2.78(.45)$ \\
\hline & & Musician & $409(49)$ & $90.7(3.1)$ & $1.6(0.8)$ & $3.57(.43)$ \\
\hline
\end{tabular}



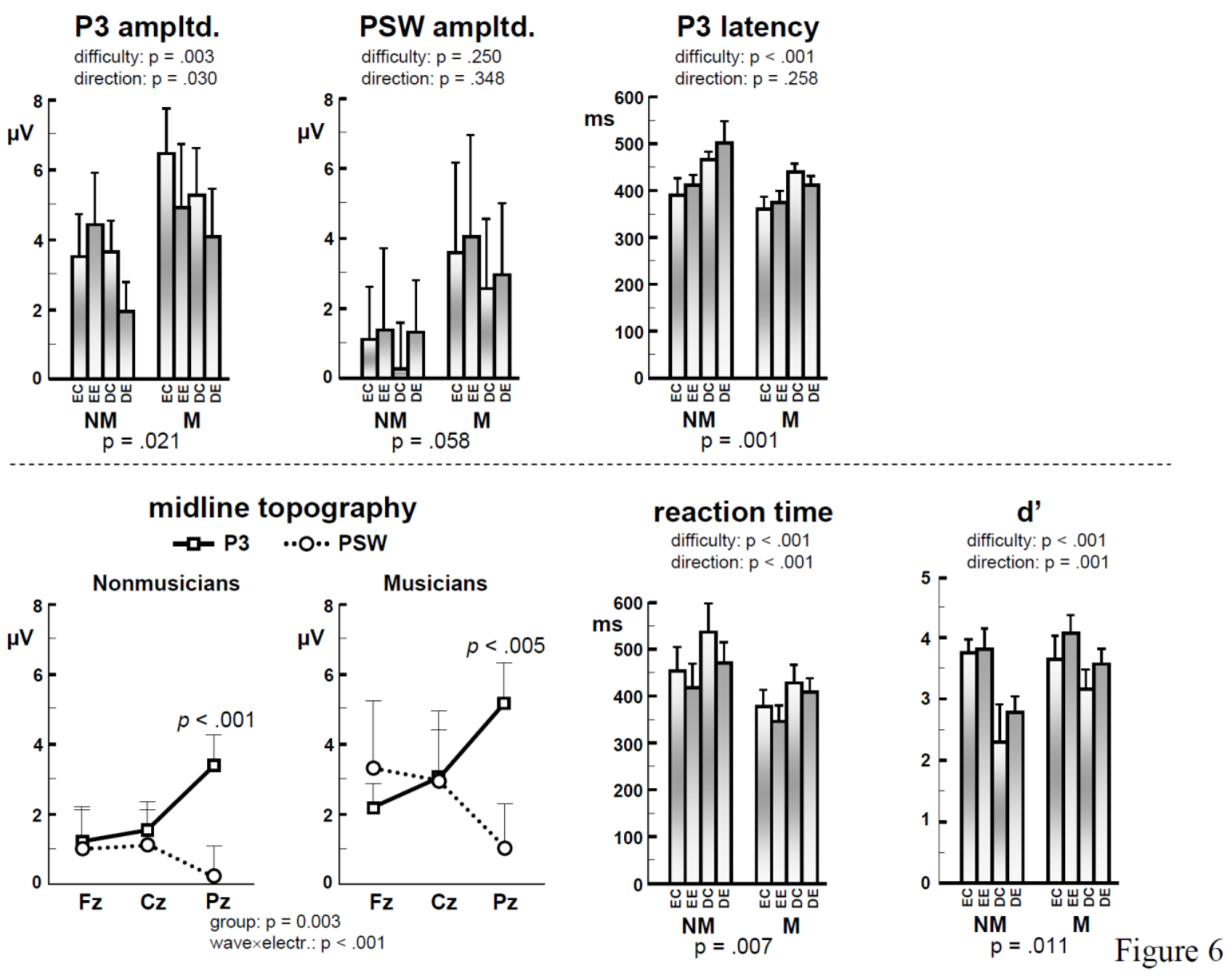

Figure 6. Statistics of the amplitudes of the parietal P3 and frontal PSW, the parietal P3 latency, the reaction-time, and the detection performance (d') that were measured from musicians $(\boldsymbol{M})$ and nonmusicians (NM) with the four types of rhythmic unit changes. $\boldsymbol{E C}$ : easyto-detect contractions; $\boldsymbol{E E}$ : easy-to-detect expansions; $\mathbf{D C}$ : difficult-to-detect contractions; $\mathbf{D E}$ : difficult-to-detect expansions. The significance levels of between-subjects and within-subject effects on each of these parameters are given as the results of separate ANOVAs. Midline 8 topographies of the P3 and PSW amplitudes in musicians and nonmusicians are also illustrated. 9 Error bars show twice the standard error of the mean.

These results show that musicians responded to RU-changes faster (about $80 \mathrm{~ms}$ earlier) than nonmusicians $[\mathrm{F}(1,22)=8.973, \mathrm{p}=.007]$. Across both groups it was observed that the easier the RU-change to detect, the faster the RT $[\mathrm{F}(1,22)=77.766, \mathrm{p}<.001]$; and, expansion type RUchanges were responded faster (about $40 \mathrm{~ms}$ earlier) than contraction type RU-changes $[F(1,22)=18.425, \mathrm{p}<.001]$ with no significant interactions between the factors. Separate ANOVAs run for the two groups showed that the differentiation difficulty and the direction of the change were significant main effects on RT in musicians [difficulty: $\mathrm{F}(1,11)=24.846$, $\mathrm{p}<.001$; change-direction: $\mathrm{F}(1,11)=13.075, \mathrm{p}=.004$ ] as well as in nonmusicians [difficulty: $\mathrm{F}(1,11)=65.031 \mathrm{p}<.001$; change-direction: $\mathrm{F}(1,11)=9,670$, $\mathrm{p}<.01]$.

Considering their average d' values well above 2, both groups were good performers in detecting the RU-changes with both detection difficulty levels. Nonetheless, the two groups had significantly different d' values $[\mathrm{F}(1,22)=7.7, \mathrm{p}=.011]$, indicating that musicians displayed even better detection performance than nonmusicians. However, this superiority of musicians over nonmusicians depended on difficulty as indicated by a significant group $\times$ difficulty interaction $[F(1,22)=11.2, p=.003]$. Separate ANOVAs were run for the two difficulty levels and it was 
found that between-subjects effect was significant for the difficult-to-detect changes $[F(1,22)=14.093, p=.001$, but not significant for the easy-to-detect changes $[F(1,23)<1]$. In other words, musicians were better performers only for the difficult-to-detect RU-changes. Across both groups it was observed that the detection performance was higher for the RU-changes that were easier to detect $[\mathrm{F}(1,22=58.2, \mathrm{p}<.001]$. Also, higher performance was noted for the changes of expansion type than those of contraction type $[\mathrm{F}(1,22)=14.9, \mathrm{p}=.001]$. However, this advantage reached statistical significance only for the difficult-to-detect expansions $[\mathrm{F}(1,22)=7.471, \mathrm{p}=.012]$. The above mentioned significant group $\times$ difficulty interaction also suggested that detection difficulty of the RU-change was a more significant factor for nonmusicians than musicians. However, separate ANOVAs that were run for each group indicated that differentiation difficulty was a significant main effect on performance (d') in nonmusicians $[\mathrm{F}(1,11)=60.777, \mathrm{p}<.001]$ as well as in musicians $[\mathrm{F}(1,11)=9.088, \mathrm{p}=.012]$.

Discriminant analysis showed that the d' obtained for difficult-to-detect RU-changes of expansion type and the RT obtained for difficult-to-detect RU-changes of contraction type are the two most important behavioral parameters (with correlation sizes of .770 and -.541, respectively, in the structure matrix) in differentiating between the members of the two groups. Using the first parameter alone, correct classification percentage was found to be $75 \%$ (Wilks' $\left.\lambda=.526 ; \chi^{2}=13.817, \mathrm{df}=1, \mathrm{p}<.001\right)$. Inclusion of both $\mathrm{d}^{\prime}$ and $\mathrm{RT}$ values obtained in all of the four experiments raised the correct classification percentage to $91.7 \%$ (Wilks' $\lambda=.397 ; \chi^{2}=16.647$, $\mathrm{df}=8, \mathrm{p}=.034$ ). These behavior-based classification results are given in Table 2 for the two cases together with the ERP-based classification results for comparison.

P3 latency was found to be significantly correlated with $R T$ in both musicians $(R=.45 ; \mathrm{p}<.001)$ and nonmusicians $(\mathrm{R}=.29 ; \mathrm{p}<.044)$.

\section{DISCUSSION}

Since we obtained the behavioral data only for their supplementary value, we will not analyze them separately but discuss together with the electrophysiological data, where appropriate. Rather than the ERPs directly recorded, we will discuss below the "deviant-standard" difference waveforms presented in Fig. 4, which contain merely the endogenous components of the targetevoked ERPs obtained from the two groups.

\section{The P3}

The main concern of the present study was to find if a change in rhythm elicits different ERPs in musicians and nonmusicians, giving special emphasis to the P3 component. On the contrary to the literature not supporting a clear effect of musical expertise on the P3 [10, 22-24], our results show with high confidence that musicians at professional level respond to rhythmic unit changes with P3s that are larger in amplitude and earlier in latency than those recorded from the control group with no formal musical training. Considering the general conviction that the latency of the P3 corresponds to the speed of cognitive processing and its amplitude shows the amount of brain energy resources allocated for this process [4], this result can be considered a normal consequence of the expected superiority of musicians in rhythm perception. This superiority the musicians must have either possessed at birth or gained through extensive musical training at professional level is in fact demonstrated in the present study by the behavioral results indicating their significantly shorter RTs and significantly higher rhythm-change detection performance (d') compared to nonmusicians. 
Both ANOVA and discriminant analysis showed that the latency of P3 is more closely correlated with musical expertise than its amplitude. This finding is in harmony with the literature reporting that P3 latency rather than P3 amplitude is associated with cognitive capability in both normal and patient populations [3]. However, musical expertise proves to be a significant main effect also on the P3 amplitude, and it seems a classification accuracy as high as $91.7 \%$ can be achieved when both latency and amplitude of the P3 are evaluated. It is interesting to see that this classification accuracy is not less than the accuracy achieved by using the behavioral parameters such as the detection performance index (d', combining the hit and false alarm rates) and RT, providing another strong evidence for the long suspected link between the ERP P3 and cognitive abilities. The remarkably high correct classification proportions reported in the present study denote, however, posterior probabilities (i.e., probabilities of the outcomes of an experiment after it has been performed) and they may not ensure the predictive validity of the model.

Jongsma et al. [21] observed that the P3 was sensitive to violation of musical congruencies in such a way that the larger the violation, the larger the amplitude of the P3. Although in our case the violation was not of a musical congruency but an expectation that was set simply by the higher probability of occurrence of standard RUs than target RUs, the P3 elicited by difficult-todetect RU-changes was recorded with significantly smaller amplitudes and longer latencies than those elicited by easy-to-detect changes. This is in line with the well known mutual relationship of the amplitude and latency of the P3 with the target/standard differentiation difficulty in an oddball task [5, 37, 38].

Concerning the behavioral responses evaluated in the present study, however, not only musicians but also nonmusicians were very good in detecting the more difficult-to-discriminate target RUs, as shown by their average d' values above 2 . This observation is parallel with that of Tervaniemi et al. [20] who reported that also nonmusicians detected quite reliably very small pitch changes. Analysis of our d' data showed that the effect of musical expertise was, nonetheless, reflected in performance as significant shortening of RT and improvement in discrimination ability. RT seemed to be affected by expertise for both levels of differentiation difficulty of the target. However, expertise proved to be a significant effect only for difficult-to-detect targets. This was probably because of a ceiling effect similar to that described by Tervaniemi et al. [20]. They also reported that superiority in pitch discrimination accuracy in musicians over nonmusicians was noted with small and medium pitch changes but not with large changes.

Considering the generally observed and above mentioned effect of difficulty on the amplitude of the P3 [5], recording significantly higher detection performance and shorter RTs with the expansion type RU-changes across both groups should mean that this type of RU-change was more readily detected than the contraction type. However, the P3 elicited by the expansion-type RU-changes had smaller amplitudes than that elicited by the RU-changes of contraction type. A possible explanation for this discrepancy may be related to the above mentioned ambiguity in the timing of "the event" to be responded in the case of expansion. Although we assumed that the target event in the expansion type RU-changes occurs physically at the onset of the second beat of the standard RU, the exact time the expectation is disconfirmed (i.e., the time the delay in second beat is sensed) cannot be defined unambiguously. In the case of target contractions, on the other hand, this time is exactly defined as the onset of the second beat in the deviant RU. Because of the ambiguity in the perceived onset time of a "non-occurrence", the variability of the P3 latency might have increased, causing a decrease in the peak amplitude of the averaged $\mathrm{P} 3$ in this case. 
Jongsma et al. [21] found that changes in tempo affected an early and a late subcomponent of P3 in participants who were able to detect very small tempo changes. They reported that accelerations in tempo were mainly characterized by an increase in the late P3 subcomponent (P3b) over parietal sites whereas decelerations were characterized by an increase in the early P3 subcomponent (P3a) over fronto-central sites. If the conceptual similarity between the events of acceleration/deceleration in their study and the events of contraction/expansion in the present study may be assumed, our findings are in partial agreement with their observations. In the contraction-type change, this ERP component was recorded with maximal amplitudes at the parietal electrode also in the present study. However, in none of our four experiments we could observe a frontal or even central dominance of the P3 in the way described by Jongsma et al. [21] for P3a. This discrepancy may result from the difference between the stimulation paradigms used in the two studies. Firstly, they did not present their stimuli using an oddball sequence. Instead, their subjects listened, in separate trials, to a number of sequences of five beats and judged whether the last target beat of the sequence was 'too early' or 'too late' in comparison with the preceding four. Since the last interval was always the same $(600 \mathrm{~ms})$, the length of the preceding intervals must have signaled whether the sequence will end with acceleration or deceleration, producing a priming effect. Secondly, the target event was in fact a change in tempo in their case but a change in regularly repeating rhythmic units in our experiments, although in our case too the occurrence of a target was perceived as speeding up or slowing down of the drum beats.

Concluding the discussion of the P3 recorded in the present study, this ERP component is probably not very much different from the typical P300 that is elicited by any violation of a simple auditory expectation based on the greater probability of occurrence of the standards. A more pronounced attention effect on P3 in musicians compared to untrained subjects [19], may also have contributed to this difference. However, it remains unanswered if this P3 modification (enhancement of its amplitude and shortening of its latency) in musicians is an index of a longterm plasticity leading to an improvement in their sensory abilities to detect temporal deviances or whether it is because their auditory working memory develops so well through extensive musical training that it can build more readily and precisely the traces or templates for the temporal structures of the standard and target rhythmic units in a fashion similar to that suggested by Pfeuty et al. [39] for tempo encoding. The latter explanation is, nonetheless, in harmony with the findings and interpretation of Crummer et al. [17] and Wayman et al. [40]. In these studies, the subjects with absolute pitch displayed the shortest mean P3 latencies, but had smaller P3 amplitudes relative to both musicians and nonmusicians. These results may suggest that the P3 is a manifestation of processing activities that are involved in "working memory" function [41], and that the enhancement of P3 amplitude and shortening of P3 latency that we observed in musicians may be because their neural system underlying this function has become highly developed through extensive training. Still another implication of the observed musician/nonmusician differences would be that the two groups might have already initially differed in some neural mechanisms related to the abilities to make fine sensory discriminations, and persons having such good abilities could have more probably entered professional music education.

\section{Positive Slow Wave (PSW)}

Although in both groups a positive slow wave (PSW) following the P3 was recorded from all the electrodes used, the PSW was observed with notably smaller amplitudes in nonmusicians (see Fig. 4). We believe that this sustained positive shift lasting up to $1400 \mathrm{~ms}$ after the RU-change can be identified with the fairly tonic late positivity recorded by Koelsch and Mulder [42] in 
response to unexpected deviant chords, and with the P600 and the later steady positivity described by Patel et al. [9], and also with the music-related late positive component (LPC) described by Besson and Faïta [18], and Regnault et al. [10].

Besson and Faita [18] showed that the LPC they recorded in response to musical incongruities was larger for musicians than nonmusicians. They further reported that when identification of the incongruity was independent of specific musical knowledge the LPC did not differ in amplitude between musicians and nonmusicians; and, when knowledge of musical rules was necessary to perform the task its amplitude was larger for musicians than nonmusicians. Regnault et al. [10], on the other hand, reported that musicians and nonmusicians did not respond with significantly different slow wave amplitudes to mismatches in consonance or harmonic context of chords, which were indeed musically relevant stimuli involving harmonic expectancy. Considering these observations, it is rather puzzling that we observed a marginal but significant difference between the PSW amplitudes recorded from musicians and nonmusicians despite the fact that the target events we used were merely very simple changes in rhythmic unit.

P3 and PSW proved to have quite different midline topographies in the present study as it is clearly seen in the ERPs in Fig. 4 and shown by the significant electrodexwave interaction displayed in Fig. 6. However, there are studies reporting that the P3 and PSW have similar scalp topographies, implying a common source, although slightly different scalp distributions were found for the principle components corresponding to these waves and somewhat different cognitive functions were associated with them $[8,43,44]$. This discrepancy could stem from the fact that PSW onset latencies may be so short as to overlap with the latency range of P3 for perceptual operations such as detection and identification of an external stimulus [6]. However, by measuring the P3 amplitudes from the high-pass filtered ERPs, we have tried to avoid superposition of the P3 and PSW amplitudes due to this temporal overlap within the latency range of $\mathrm{P} 3$. We believe it was due to this measure and because a time window well beyond the P3 latency range was used for assessing the PSW magnitude that we could uncover the otherwise not noticeable topographical difference between the P3 and PSW. This point can be demonstrated on the topographical plot in Fig. 6 where one can see that superposition of these two waves at $\mathrm{Pz}$ would have decreased the topographical contrast between them and masked the actually quite significant electrodexwave interaction.

As was briefly reviewed by Ruchkin et al. [6], the parameters of slow waves (their amplitude, onset latency, polarity, and topography) may greatly vary depending on the experimental design and the task given to the subject. Parallel to this fact, these waves are associated in literature with diverse cognitive functions such as memory storage, retrieval and updating, rule learning, perceptual operations, scanning and mental imagery, sustained attention, decision making, response selection, completion of a task, etc. We refrain, however, from speculating further on the possible associations of the PSW we measured, and merely state that this late positive component seems to be affected by musical expertise and can be used as an additional parameter indexing the level of musical abilities of a group of subjects.

\section{CONCLUSION}

Unlike the results of a few rhythm-related ERP P3 studies in literature, our study has shown with high statistical confidence that occasional rhythm changes elicited P3s with larger amplitudes and shorter latencies in musicians than in nonmusicians. Also the positive slow wave (PSW) elicited frontally had notably larger amplitudes in musicians than nonmusicians, though the 
difference was marginally significant. These two waves were shown to have significantly different scalp midline topographies suggesting that their generator locations and psychoanatomical associations should be different from each other. Interestingly, similar musician vs. nonmusician ERP differences to those reported in previous studies for violations of relatively complex musical regularities could also be observed with very simple rhythmic unit alterations.

Based on the generally accepted functional associations of the P3 and PSW, our finding that these waves have larger amplitudes in musicians should indicate that the amount of energy resources allocated in their brain for the neural mechanisms participating in rhythm perception is larger than that in nonmusicians. Our finding that the P3 latency is shorter for musicians, on the other hand, should be a consequence of the fact that these cognitive mechanisms work faster in musicians, as also suggested by their shorter RTs. These conclusions should also be valid even if the $\mathrm{P} 3$ does not directly reflect cognitive processing or target stimulus identification in psychological terms, and rather originate from a closure mechanism which ends the activation of the decisive machinery as stated by Desmedt [45] and Verleger [46]. This is because termination of a process that has required a larger amount of energy resources and a shorter running time will most likely be accompanied also by a larger and earlier deflection in EEG.

However, considering the results of a recent study in which musicians displayed larger MMN and $\mathrm{N} 2 \mathrm{~b}$ than nonmusicians not only for music but also for speech sounds during attentional listening [47], specificity of ERP indices for a certain discriminative ability should be argued with care. Therefore, the fact that the stimuli we employed were occasional rhythmic unit alterations does not assure the specificity of these ERP indices for rhythm perception; they may well indicate a sensory and/or cognitive superiority of musicians over nonmusicians in more general terms. This specificity issue remains to be resolved in future studies which should include appropriate control recordings made with adequately high spatial resolution. Also, our crosssectional study may not conclusively prove that the observed ERP differences have a causal relationship with training. This issue will be dealt with in our future studies which have already been planned to follow a longitudinal approach employing a "pre versus post" design.

Musical expertise proved to be a statistically significant main effect on the ERP parameters analyzed, and the P3 latency appeared to be the parameter that indicated with highest confidence the between-groups differences. The one next to it was the amplitude of the same wave. The results of discrimination analyses also supported this finding; a classification accuracy of above $90 \%$ could be achieved when both parameters were used together in a discrimination model. And, this accuracy proved to be at least as good as the accuracy attained by using the behavioral indices for the same classification. This close parallelism of the electrophysiological indices with the behavioral ones clearly indicate, ones more, the fact that the ERP P3 does have a connection to cognitive behavior.

\section{REFERENCES}

1. Kutas M, McCarthy G, Donchin E (1977)Augmenting mental chronometry: the P300 as a measure of stimulus evaluation time. Science 197:792-795.

2. Antal A, Keri S, Kovacs, G, Janka Z, Benedek G (2000) Early and late components of visual categorisation: an event-related potential study. Cogn Brain Res 9:117-119.

3. Polich J (1998) P300 Clinical Utility and Control of Variability. J Clin Neurophysiol 15:1433. 
4. Kok A (1997) Event-related potentials (ERP) reflections of mental resources: a review and synthesis. Biol Psychol 45:19-56.

5. Polich J (2007) Updating P300: An integrative theory of P3a and P3b. Clin Neurophysiol 118: 2128-2148.

6. Ruchkin DS, Sutton S (1983) Positive slow wave and P300: Association and disassociation. In: Gaillard AKW, Ritter W (eds) Tutorials in ERP Research: Endogenous Components, North Holland, Amsterdam, pp 233-250.

7. Ruchkin DS, Johnson R, Mahaffey D, Sutton S (1988) Toward a functional categorization of slow waves. Psychophysiol 25:339-353.

8. García-Larrea L, Cézanne-Bert G (1998) P3, Positive slow wave and working memory load: a study on the functional correlates of slow wave activity. Electroenceph clin Neurophysiol 108:260-273.

9. Patel AD, Gibson E, Ratner J, Besson M, Holcomb PJ (1998) Processing syntactic relations in language and music: an event-related potential study. J Cogn Neurosci 10:717-733.

10. Regnault P, Bigand E, Besson M (2001) Different brain mechanisms mediate sensitivity to sensory consonance and harmonic context: evidence from auditory event-related brain potentials. J Cogn Neurosci 13:241-255.

11. Donchin E, Coles MGH (1988) Is the P300 component a manifestation of context updating? Behav Brain Sci 11:355-572.

12. Ilan AB, Polich J (1999) P300 and response time from a manual Stroop task. Clin Neurophysiol 110:367-373.

13. Pantev C, Oostenveld R, Engelien A, Ross B, Roberts LE, Hoke M (1998) Increased auditory cortical representation in musicians. Nature 392:811-814.

14. Pantev C, Engelien A, Cadia V, Elbert T (2001) Representational cortex in musicians. Ann NY Acad Sci 930:300-304.

15. Münte TF, Altenmüller E, Jäncke L (2002) The musician's brain as a model of neuroplasticity. Nature Rev Neurosci 3:473-478.

16. Jäncke L (2009) The plastic human brain. Restor Neurol Neurosci 27:521-538.

17. Crummer GC, Walton JP, Wayman JW, Hantz EC, Frisina RD (1994) Neural processing of musical timbre by musicians, nonmusicians, and musicians possessing absolute pitch. J Acoust Soc Am 95:2720-2727.

18. Besson M, Faïta F (1995) An event-related potential (ERP) study of musical expectancy: Comparison of musicians with nonmusicians. J Exp Psychol: Hum Percept Performance 21:1278-1296.

19. Rüsseler J, Altenmüller E, Nager W, Kohlmetz C, Münte TF (2001) Event-related brain potentials to sound omissions differ in musicians and non-musicians. Neurosci Lett 308:33-36. 
20. Tervaniemi M, Just V, Koelsch S, Widmann A, Schröger E (2005) Pitch discrimination accuracy in musicians vs nonmusicians: an event-related potential and behavioral study. Exp Brain Res 161:1-10

21. Jongsma MLA, Meeuwissen E, Vos PG, Maes R (2007) Rhythm perception: Speeding up or slowing down affects different subcomponents of the ERP P3 complex. Biol Psychol 75: 219228

22. Koelsch S, Schmidt B-H, Kansok J (2002) Effects of musical expertise on the early right anterior negativity: an event-related brain potential study. Psychophysiol 39:657-663.

23. Jongsma MLA, Quian Quiroga R, van Rijn CM (2004) Rhythmic training decreases latencyjitter of omission evoked potentials (OEPs). Neurosci Lett 355 :189-192.

24. Jongsma MLA, Desain P, Honing H (2004) Rhythmic context influences the auditory evoked potentials of musicians and nonmusicians. Biol Psychol 66:129-152.

25. Brochard R, Abecasis D, Potter D, Ragot R, Drake C (2003) The "ticktock" of our internal clock: direct brain evidence of subjective accents in isochronous sequences. Psychol Sci 14:362366.

26. Vuust P, Pallesen KJ, Bailey C, van Zuijen TL, Gjedde A, Roepstorff A, Østergaard L (2005) To musicians, the message is in the meter: pre-attentive neuronal responses to incongruent rhythm are left-lateralized in musicians. Neuroimage 24:560-564.

27. Jongsma ML, Eichele T, Quian Quiroga R, Jenks KM, Desain P, Honing H, van Rijn CM (2005) Expectancy effects on omission evoked potentials in musicians and nonmusicians. Psychophysiol 42:191-201.

28. Näätänen R (1992) Attention and Brain Function. Erlbaum, Hillsdale NJ, pp 236-244.

29. Durr W, Gerstenberg W, Harvey J (1980) Rhythm. In: Sadie S, editor. The New Grove Dictionary of Music and Musicians. Macmillan Publishers Ltd, London, pp 804-824.

30. Fraisse P (1982) Rhythm and tempo. In: Deutsch D, editor. The Psychology of Music, Academic Press, New York, pp 149-180.

31. Krumhansl CL (2000) Rhythm and pitch in music cognition. Psychol Bull 126:159-179.

32. Garner WR (1974) The Processing of Information and Structure. Erlbaum, Potomau, MD.

33. Kristofferson $A B$ (1980) A quantal step function in duration discrimination. Perception Psychophysics 27:300-306.

34. Gonsalvez CJ, Polich J (2002) P300 amplitude is determined by target-to-target interval. Psychophysiol 39:388-396.

35. Croft RJ, Gonsalvez CJ, Gabriel C, Barry RJ (2003) Target-to-target interval versus probability effects on P300 in one- and two-tone tasks. Psychophysiol 40:322-328 
36. Wickens TD (2001) Elementary Signal Detection Theory. Oxford Univ Press, London, p. 288.

37. Isreal JB, Chesney GL, Wickens CD, Donchin E (1980) P300 and tracking difficulty: evidence for multiple resources in dual-task performance. Psychophysiol 17:259-273.

38. Polich J (1987) Task difficulty, probability, and inter-stimulus interval as determinants of P300 from auditory stimuli. Electroenceph clin Neurophysiol 68:311-320.

39. Pfeuty M, Ragot R, Pouthas V (2003) Processes involved in tempo perception: A CNV analysis. Psychophysiol 40:69-76.

40. Wayman JW, Frisina RD, Walton JP, Hantz EC, Crummer GC (1992) Effects of musical training and absolute pitch ability on event-related activity in response to sine tones. $\mathrm{J}$ Acoust Soc Am 91:3527-531.

41. Klein M, Coles MGH, Donchin E (1984) People with absolute pitch process tones without producing a P300. Science 223:1306-1309.

42. Koelsch S, Mulder J (2002) Electric brain responses to inappropriate harmonies during listening to expressive music. Clin Neurophysiol 113:862-869.

43. Squires N, Squires K, Hillyard S (1975) Two varieties of long-latency positive waves evoked by unpredictable auditory stimuli in man. Electroenceph clin Neurophysiol 38:387-401.

44. Honda M, Barrett G, Yoshimura N, Ikeda A, Nagamine T, Shibasaki H (1996) Event-related potentials during paired associative memory paradigm. Electroenceph clin Neurophysiol 100:407-421.

45. Desmedt JE (1980) P300 in serial tasks: An essential post-decision closure mechanism. Prog Brain Res 54:682-686.

46.Verleger R (1988) Event-related potentials and cognition: A critique of the context updating hypothesis and an alternative interpretation of P3. Behav Brain Sci 11:343-427.

47. Tervaniemi M, Kruck S, De Baene W, Schröger E, Alter K, Friederici AD (2009) Top-down modulation of auditory processing: effects of sound context, musical expertise and attentional focus. Eur J Neurosci 30:1636-1642. 